\title{
Body weight and glucose metabolism have a different effect on circulating levels of ICAM-1, E-selectin, and endothelin-1 in humans
}

Antonio E Pontiroli, Pierluigi Pizzocri, Diana Koprivec, Paola Vedani ${ }^{1}$, Monica Marchi ${ }^{1}$, Cinzia Arcelloni $^{2}$, Rita Paroni ${ }^{2}$, Katherine Esposito ${ }^{3}$ and Dario Giugliano ${ }^{3}$

Cattedra di Medicina Interna, Università degli Studi, 2nd Divisione di Medicina Interna, Ospedale San Paolo, Italy, ${ }^{1}$ Divisione di Medicina Interna and ${ }^{2}$ Laboratorio Tecniche Separative, IRCCS Ospedale San Raffaele and Ateneo Vita-Salute, Milano, Italy and ${ }^{3}$ Divisione di Malattie Metaboliche, 2nd Università degli Studi, Napoli, Italy

(Correspondence should be addressed to Antonio E Pontiroli, Ospedale San Paolo, Via A. di Rudinì 8, 20142 Milano, Italy; Email: antonio.pontiroli@unimi.it)

\begin{abstract}
Background: Endothelial dysfunction and inflammation are present in both type 2 diabetes mellitus (T2DM) and obesity. In this paper we compared the role of weight loss and of glycaemic control in determining circulating levels of ICAM-1, endothelin-1 (ET-1), and E-selectin in patients with morbid (grade 3) obesity.

Methods and Results: ICAM-1, E-selectin, and ET-1 were higher in obese patients $(n=96)$ than in lean controls $(n=30)$; among obese patients, the three molecules were higher in T2DM patients $(n=26)$ than in patients with normal (NGT, $n=43$ ) or impaired (IGT, $n=27$ ) glucose tolerance. Sixty-eight obese patients had a significant weight loss induced by bariatric surgery, and showed a significant decrease in blood glucose, HbA1c and all molecules, so that ICAM-1, E-selectin, and ET-1 were not different in NGT, IGT and T2DM patients, and in lean controls; in 13 patients with a small weight loss induced by diet, changes were not significant, in spite of a significant reduction in blood glucose and $\mathrm{HbA1c}$. At stepwise regression, changes in ICAM-1, ET-1, and E-selectin significantly correlated only with change in body mass index.

Conclusions: These data indicate that weight loss is more important than glycaemic control in regulating circulating levels of ICAM-1, ET-1, E-selectin in morbidly obese subjects.
\end{abstract}

European Journal of Endocrinology 150 195-200

\section{Introduction}

Endothelial dysfunction and inflammation, as indicated by abnormal flow-dependent vasodilation and by increased circulating levels of adhesion molecules (ICAM-1 and E-selectin) $(1,2)$ are known to occur in type 2 diabetes mellitus (T2DM), and allegedly predict systemic atherogenesis (3). In addition, both hyperglycaemia and insulin administration increase circulating levels of endothelin-1 (ET-1), a potent vasoconstrictor peptide $(4,5)$. However, the majority of patients with T2DM are obese, and this raises the question of the importance of diabetes per se and of obesity per se. In fact, endothelium-dependent vasodilation is reduced in obesity $(6,7)$; in addition, ET-1 release can be elicited in lean subjects by co-administration of insulin and triglycerides, and is increased in non-diabetic obese subjects with 'metabolic syndrome' (8). Finally, neither short-term nor long-term improvements in glycaemic control lead to changes in adhesion molecules in obese T2DM patients, suggesting that obesity is more important than glycaemic control in determining circulating levels of ICAM-1, ET-1 and E-selectin $(9,10)$. The aim of this study was to compare the effect of a sustained and persistent weight loss and of good metabolic control on circulating levels of ICAM-1, ET-1, and Eselectin in morbidly obese patients.

\section{Materials and methods}

\section{Basal evaluations}

Laparoscopic adjustable gastric banding (LAGB) is an on-going program approved by the local Ethics Committee at the IRCCS Ospedale San Raffaele and at Ospedale San Paolo since June 1996 (11), in patients with morbid (grade 3) obesity according to World Health Organisation (WHO) criteria (12). We considered 96 consecutive patients entering the program, recruited locally or referred from the Divisione di Malattie 
Metaboliche, Università degli Studi di Napoli; inclusion criteria were age 18 to 65 years inclusive, body mass index $\left(\mathrm{BMI}, \mathrm{kg} / \mathrm{m}^{2}\right)>40.0$, or $>35.0$ in the presence of co-morbidities (T2DM or arterial hypertension) (13), history of at least two previous attempts to lose weight with dietary and medical measures followed by relapse of obesity; exclusion criteria were: obesity secondary to endocrinopathies (Cushing's disease or syndrome, hypothyroidism), gastrointestinal inflammatory diseases, risk of upper gastrointestinal bleeding, pregnancy, alcohol or drug addiction, previous or current malignancies, myocardial infarction or unstable angina during the preceding 6 months (13). Waist circumference was measured as an index of body fat distribution (14). Two oral glucose tolerance tests (OGTT, $75 \mathrm{~g}$ ) were performed in all patients (15). A complete evaluation of micro- and macroangiopathic complications was performed in patients with T2DM (usually the diagnosis was the result of OGTT). Retinal photography revealed absence of retinopathy in all patients; microalbuminuria and proteinuria were absent in all patients, and ECG and medical history revealed no signs/symptoms of coronary heart disease. Blood pressure was measured by the same physician, using the same sphygmomanometer with an appropriate cuff. Patients were considered hypertensive when systolic/diastolic blood pressure was $>140 / 90 \mathrm{mmHg}$ or when patients were under stable anti-hypertensive medication. No T2DM patient was receiving insulin, and only 5/26 patients were receiving metformin, $850 \mathrm{mg}$ b.i.d. As part of the program, obese patients underwent gastroscopy and a full blood examination, including markers of inflammation such as erythrocyte sedimentation rate (ESR) and white blood cells.

\section{Control subjects}

Thirty healthy lean volunteers with a normal glucose tolerance were recruited from the medical staff of the Divisione di Malattie Metaboliche, Università degli Studi di Napoli and underwent the same anthropometric and biochemical evaluations as the obese patients.

\section{Follow-up evaluations}

For the purpose of this study we considered 68 subjects (36 with normal glucose tolerance (NGT), 23 with impaired glucose tolerance (IGT), 9 T2DM) undergoing LAGB and 13 subjects (7 NGT, 4 IGT, 2 T2DM) refusing LAGB and followed-up with a traditional dietary treatment. After LAGB, the suggested diet was $1000 / 1100 \mathrm{kcal} /$ day in women and men respectively, and included $48 \%$ carbohydrates (starch or bread), $33 \%$ proteins (fat free parts of different animals and fishes) and 19\% lipids (olive oil). Diets were given by a dietitian and a physician with specific training in obesity treatment and nutrition; iron was supplemented on the basis of blood examinations performed during the second month. Patients were also advised to take $30 \mathrm{~min}$ physical aerobic activity every day to avoid muscular loss, with a gradual increase over a onemonth period. Physical activity could be gymnastic, swimming or dancing. Patients were scheduled for further assessment at three-month intervals for one year. Diet-treated patients were evaluated under basal conditions and at three-month intervals for one year, and their diet was 1000/1200 Kcal/day for women and men respectively (49\% carbohydrates, 29\% proteins, $22 \%$ lipids).

\section{Laboratory methods}

Patients were studied supine in the morning, during one of the two OGTTs, after an overnight fast and at least $1 \mathrm{~h}$ of bed rest. An i.v. 20-gauge cannula was inserted in the left antecubital vein, kept patent by a slow infusion of $0.9 \% \mathrm{NaCl}$ solution. Specimens for ET-1 were collected in pre-chilled EDTA tubes with $200 \mu$ l aprotinin, and specimens for E-selectin and ICAM-1 were collected in pre-chilled tubes with no additives, centrifuged at $4{ }^{\circ} \mathrm{C}$ and stored at $-70^{\circ} \mathrm{C}$. Blood glucose levels were measured by a glucose-oxidase method (YSI, Yellow Springs, OH, USA). Insulin was assayed by a Mycroparticle Enzyme Immunoassay (MEIA, IMX, Abbott Laboratories, Abbott Park, IL, USA) with a monoclonal antibody without cross-reactivity with human pro-insulin; sensitivity was $6.0 \mathrm{pmol} / \mathrm{l}$; intra-assay coefficient of variation $(\mathrm{CV})$ was $3.0 \%$, interassay $\mathrm{CV}$ was $5.0 \%$. HbA1c was assayed by a routine HPLC method (16). ET-1 was assayed by a solid-phase monoclonal antibody-based enzyme-linked immunosorbent assay (ELISA) designed to measure ET-1 in extracted EDTA plasma (Human Endothelin-1 Immunoassay, R\&D Systems, Minnieapolis, MN, USA). Briefly, to $1 \mathrm{ml}$ plasma, $1.5 \mathrm{ml}$ of a mixture of acetone: $1 \mathrm{M} \mathrm{HCl}$ :water (40:1:5, $\mathrm{v}: \mathrm{v}: \mathrm{v})$ were added. After mixing by inversion and centrifugation $\left(20 \mathrm{~min}\right.$ at $2000 \mathrm{~g}$ at $\left.4^{\circ} \mathrm{C}\right)$, the supernatant was decanted and dried in a centrifugal evaporator at $37^{\circ} \mathrm{C}$. The pellet was reconstituted with $0.25 \mathrm{ml}$ sample diluent and immediately assayed by ELISA technique following the manufacturer's instructions. ICAM1 and E-selectin were assayed by the Human soluble ICAM-1 Immunoassay and by the Human soluble ESelectin Immunoassay kits (R\&D Systems), solid-phase ELISA kits designed to measure the above mentioned parameters directly on serum samples (17-19); interand intra-assay CV were between 4.3 and 7.6 for all molecules. The homostasis model assessment (HOMA) index was calculated as insulin $(\mu \mathrm{U} / \mathrm{ml})$.blood glucose $(\mathrm{mmol} / \mathrm{l}) \cdot 22.5^{-1}(20)$.

\section{Sample size}

The sample size allowed detection of $20 \%$ difference or more between obese and control subjects in the mean 
level of ICAM-1, with a type 1 error of 0.05 and a power of 0.8. Assuming an ICAM-1 mean level of $250 \mathrm{ng} / \mathrm{ml}$, a standard deviation of $44 \mathrm{ng} / \mathrm{ml} \mathrm{(10),}$ and a rate of obese/control of 4 , a number of at least 40 obese and 8 control subjects was required.

\section{Calculations and statistical analysis}

The difference among groups for all molecules under study and for clinical, endocrine and metabolic variables was assessed using the analysis of variance. Differences between groups were assessed using the Student's $t$-test for paired or unpaired samples. Significance of multiple comparisons was adjusted by the Bonferroni correction. Because normality had not been verified on all original variables, log-transformation of data was applied, when necessary. Fisher's exact test was used to compare absolute frequencies. Pairwise correlations between adhesion molecules and anthropometric and biochemical variables, and between their changes at one year, were also calculated. Stepwise regression analysis was further carried out to estimate the independent contribution of selected variables (variables significant at linear regression plus age, sex, type of intervention) on ICAM-1, E-selectin, and ET-1, and on their changes. $P$ levels $<0.05$ were considered statistically significant.

\section{Results}

Table 1 shows clinical, endocrine, and metabolic variables (means \pm S.E.) in lean controls and in obese subjects; lean controls differed from obese subjects for all variables, except for age and sex; T2DM obese subjects differed from NGT and IGT obese subjects for HbA1c, ICAM-1, E-selectin, and ET-1; finally, obese NGT, IGT, and T2DM subjects showed progressively higher glucose levels. No obese subject had abnormal gastroscopy or inflammation markers, and no differences were found between men and women in ICAM-1, Eselectin or ET-1. Of 96 obese patients, 15 T2DM patients did not undergo the follow-up study; these two groups of T2DM patients did not differ for any clinical or metabolic variable.

After 1 year the 68 patients undergoing LAGB had a greater weight loss $\left(\mathrm{BMI}=-8.2 \pm 0.61 \mathrm{~kg} / \mathrm{m}^{2}\right)$ than diet-treated patients $\left(\mathrm{BMI}=-0.4 \pm 1.04 \mathrm{~kg} / \mathrm{m}^{2}\right)$ $(P<0.01)$, while weight loss was not statistically significant at 3 and 6 months (not shown); Table 2 shows that in the LAGB group all variables significantly declined, including ICAM, E-selectin, and ET-1, with no differences among NGT, IGT, and T2DM subjects (not shown); in the second group, only glucose and HbA1c decreased, with no change in ICAM-1, E-selectin and ET-1; the behaviour of BMI, waist circumference, arterial blood pressure, insulin, HOMA, ICAM-1, E-selectin and ET-1 was significantly different in LAGB-treated as compared with diet-treated subjects $(P<0.05$ to $P<0.001)$. After slimming, the 68 obese subjects still differed from lean controls for BMI, waist circumference and HbAlc, while ICAM-1, E-selectin and ET-1 were not different.

Table 3 shows that under basal conditions ICAM-1, ET-1 and E-selectin correlated with HbA1c, HOMA, insulin, blood glucose and BMI; at stepwise regression the three molecules correlated with $\mathrm{HbA1c}$, and ICAM-1 and E-selectin also correlated with HOMA. After 1 year, ICAM-1 correlated with insulin and HOMA, and E-selectin correlated with HOMA, HbA1c, insulin and BMI; at stepwise regression, both ICAM-1 and E-selectin only correlated with HOMA. Change in ICAM-1 and E-selectin correlated with change in BMI, and change in ICAM-1 and E-selectin also correlated with change in HOMA and insulin; at stepwise regression, change in the three molecules only correlated with change in BMI.

Table 1 Clinical, endocrine and metabolic variables evaluated in control (lean) and obese subjects with normal (NGT) or abnormal (IGT) glucose tolerance, or T2DM under basal conditions. Results are means \pm s. $\mathrm{E}$. or absolute frequencies; $n=$ number of subjects.

\begin{tabular}{|c|c|c|c|c|}
\hline & \multirow{2}{*}{$\frac{\text { Lean }}{\text { NGT }(n=30)}$} & \multicolumn{3}{|c|}{ Obese } \\
\hline & & $\mathrm{NGT}(n=43)$ & IGT $(n=27)$ & $\operatorname{T2DM}(n=26)$ \\
\hline Age (years) & $38.0 \pm 1.22$ & $39.2 \pm 1.37$ & $45.7 \pm 1.74$ & $43.6 \pm 1.46$ \\
\hline Gender (male/female) & $12 / 18$ & $10 / 33$ & $9 / 18$ & $10 / 16$ \\
\hline Arterial hypertension & $0^{*}$ & 16 & 9 & 12 \\
\hline $\mathrm{BMI}\left(\mathrm{kg} / \mathrm{m}^{2}\right)$ & $22.5 \pm 0.21^{\star}$ & $44.5 \pm 1.09$ & $44.9 \pm 1.32$ & $42.9 \pm 1.21$ \\
\hline Waist circumference $(\mathrm{cm})$ & $82.1 \pm 0.41^{*}$ & $124.7 \pm 2.49$ & $125.9 \pm 2.41$ & $122.0 \pm 2.29$ \\
\hline $\mathrm{HbA1c}(\%)$ & $3.9 \pm 0.09^{\star}$ & $6.0 \pm 0.11$ & $6.6 \pm 0.22$ & $8.0 \pm 0.32 \dagger$ \\
\hline Blood glucose (mmol/l) & $5.3 \pm 0.07^{*}$ & $5.5 \pm 0.11$ & $6.3 \pm 0.20$ & $8.2 \pm 0.31^{* *}$ \\
\hline Insulin (pmol/l) & $63.9 \pm 2.63^{\star}$ & $127.4 \pm 15.75$ & $107.1 \pm 10.99$ & $109.1 \pm 8.13$ \\
\hline HOMA§ & $2.1 \pm 0.11^{*}$ & $4.6 \pm 0.68$ & $4.6 \pm 0.54$ & $5.7 \pm 0.49$ \\
\hline ICAM-1 (ng/ml) & $255.0 \pm 7.98^{\star}$ & $300.9 \pm 11.67$ & $309.2 \pm 15.88$ & $372.3 \pm 15.63 \dagger$ \\
\hline E-selectin (ng/ml) & $39.6 \pm 1.31^{*}$ & $58.0 \pm 4.99$ & $62.0 \pm 5.42$ & $91.1 \pm 8.06 \dagger$ \\
\hline ET-1 (pg/ml) & $0.94 \pm 0.05^{\star}$ & $1.27 \pm 0.09$ & $1.12 \pm 0.09$ & $1.61 \pm 0.08 \dagger$ \\
\hline
\end{tabular}

$\S$ Insulin $(\mu \mathrm{U} / \mathrm{ml})$.glucose $(\mathrm{mmol} / \mathrm{l}) \cdot 22 \cdot 5^{-1}$.

Lean vs obese subjects: ${ }^{\star} P<0.001$. Obese subjects: NGT vs IGT vs T2DM: ${ }^{\star} P<0.001 ;$ T2DM vs NGT and IGT: $† P<0.001$. 
Table 2 Clinical, endocrine and metabolic changes at one year in obese subjects undergoing laparoscopic gastric banding (LAGB) and in subjects treated with diet. Means \pm S.E.

\begin{tabular}{|c|c|c|c|c|c|}
\hline & \multicolumn{2}{|c|}{ Group $1(\mathrm{LAGB})(n=68)$} & \multicolumn{2}{|c|}{ Group 2 (no-LAGB) $(n=13)$} & \multirow{2}{*}{$\begin{array}{c}P \text { value group } 1 \mathrm{vs} \\
\text { group } 2\end{array}$} \\
\hline & Before & After & Before & After & \\
\hline $\mathrm{BMI}\left(\mathrm{kg} / \mathrm{m}^{2}\right)$ & $45.5 \pm 0.79$ & $37.1 \pm 0.68^{\star}$ & $41.4 \pm 2.54$ & $41.0 \pm 2.21$ & $<0.001$ \\
\hline Waist circumference $(\mathrm{cm})$ & $125.7 \pm 1.69$ & $109.3 \pm 1.66^{\star}$ & $121.3 \pm 2.15$ & $120.5 \pm 2.49$ & $<0.01$ \\
\hline Systolic BP (mmHg) & $132.7 \pm 1.82$ & $126.3 \pm 1.56^{\star}$ & $137.5 \pm 3.77$ & $142.1 \pm 3.17$ & $<0.05$ \\
\hline Diastolic BP (mmHg) & $84.1 \pm 1.15$ & $79.7 \pm 1.24^{\star}$ & $86.2 \pm 1.52$ & $91.7 \pm 2.91$ & $<0.05$ \\
\hline $\mathrm{HbA} 1 \mathrm{c}(\%)$ & $6.4 \pm 0.15$ & $5.6 \pm 0.09^{\star}$ & $6.6 \pm 0.62$ & $5.7 \pm 0.65^{\star}$ & NS \\
\hline Insulin (pmol/l) & $128.1 \pm 10.57$ & $70.1 \pm 4.76^{\star}$ & $94.5 \pm 9.52$ & $97.5 \pm 9.47$ & $<0.01$ \\
\hline Blood glucose (mmol/l) & $6.3 \pm 0.18$ & $5.3 \pm 0.11^{\star}$ & $5.9 \pm 0.42$ & $4.9 \pm 0.35^{\star}$ & NS \\
\hline HOMA§ & $5.1 \pm 0.28$ & $2.4 \pm 0.18^{\star}$ & $3.5 \pm 0.36$ & $3.3 \pm 0.52$ & $<0.01$ \\
\hline ICAM-1 (ng/ml) & $322.6 \pm 10.68$ & $274.3 \pm 6.79^{\star}$ & $258.4 \pm 15.32$ & $280.1 \pm 14.87$ & $<0.01$ \\
\hline E-selectin (ng/ml) & $65.5 \pm 4.50$ & $42.4 \pm 2.12^{\star}$ & $56.6 \pm 8.64$ & $53.9 \pm 4.32$ & $<0.01$ \\
\hline ET-1 (pg/ml) & $1.31 \pm 0.07$ & $1.10 \pm 0.05^{\star}$ & $1.04 \pm 0.09$ & $1.21 \pm 0.13$ & $<0.01$ \\
\hline
\end{tabular}

$\S$ Insulin $(\mu \mathrm{U} / \mathrm{ml})$.glucose $(\mathrm{mmol} / \mathrm{l}) \cdot 22.5^{-1}$

Before vs after: ${ }^{\star} P<0.01$. NS, not significant.

\section{Discussion}

In this paper we found that obese subjects with T2DM have higher ICAM-1, E-selectin and ET-1 levels than those seen in obese NGT or IGT subjects, in spite of similar BMI and waist circumference measurements; in addition, ICAM-1, E-selectin, and ET-1 levels correlated with blood glucose and HbA1c; all these data indicate the importance of the glycaemic milieu in determining circulating levels of ICAM-1, E-selectin and ET-1 (1, 2); however, the three molecules were significantly higher in obese subjects as a group than in lean controls, and also correlated with BMI, HOMA and insulin; at stepwise regression ET-1 correlated with $\mathrm{HbAlc}$, and ICAM-1 and E-selectin also correlated with HOMA. Therefore, we should assume that obesity and the glycaemic milieu are at least equally important in determining circulating levels of the three molecules $(6-8)$.

In trying to dissect out the role of glycaemic milieu and of body weight, in the prospective study we analysed separately LAGB-treated and diet-treated patients and found that significant weight loss is accompanied by a significant decrease in HbAlc and in ICAM, Eselectin and ET-1; in diet-treated patients who only obtained improvements in blood glucose and HbAlc and had no decrease in body weight, no change in ICAM, E-selectin, and ET-1 was observed. In the 68 patients with a significant weight loss, ICAM-1, ET-1 and E-selectin were not different from lean controls in spite of differences in BMI, waist circumference and $\mathrm{HbA1c}$. In contrast to what happened under basal conditions when the three molecules correlated with

Table 3 Correlations (linear regression and stepwise regression) between ICAM-1, ET-1 and E-selectin (dependent variables, logtransformed) and selected anthropometric and metabolic indexes. In A and B correlations are between absolute values; in $C$ correlations are between changes. Only significant regressions are indicated.

\begin{tabular}{|c|c|c|c|c|c|c|c|c|c|c|}
\hline \multirow{3}{*}{$\begin{array}{l}\text { Dependent } \\
\text { variable }\end{array}$} & \multirow{3}{*}{$\begin{array}{c}\text { Independent } \\
\text { variable }\end{array}$} & \multicolumn{3}{|c|}{ A: under basal conditions } & \multicolumn{3}{|c|}{ B: follow-up } & \multicolumn{3}{|c|}{ C: changes $(\Delta)$} \\
\hline & & \multicolumn{2}{|c|}{$\begin{array}{l}\text { Linear } \\
\text { regression }\end{array}$} & \multirow{2}{*}{$\begin{array}{c}\text { Stepwise } \\
\text { regression } \\
P\end{array}$} & \multicolumn{2}{|c|}{$\begin{array}{l}\text { Linear } \\
\text { regression }\end{array}$} & \multirow{2}{*}{$\begin{array}{c}\text { Stepwise } \\
\text { regression } \\
P\end{array}$} & \multicolumn{2}{|c|}{$\begin{array}{l}\text { Linear } \\
\text { regression }\end{array}$} & \multirow{2}{*}{$\begin{array}{c}\text { Stepwise } \\
\text { regression } \\
P\end{array}$} \\
\hline & & r & $P$ & & r & $P$ & & $r$ & $P$ & \\
\hline \multirow[t]{5}{*}{ ICAM-1 } & $\mathrm{HbA1c}$ & 0.41 & 0.0001 & 0.001 & & & & & & \\
\hline & HOMA & 0.29 & 0.0011 & 0.011 & 0.29 & 0.01 & 0.01 & 0.24 & 0.0387 & \\
\hline & Insulin & 0.21 & 0.0193 & & 0.26 & 0.0253 & & 0.26 & 0.0206 & \\
\hline & Glucose & 0.39 & 0.0001 & & & & & & & \\
\hline & BMI & 0.23 & 0.0111 & & & & & 0.30 & 0.0073 & 0.01 \\
\hline \multirow[t]{5}{*}{ ET-1 } & $\mathrm{HbA1c}$ & 0.47 & 0.0001 & 0.001 & & & & & & \\
\hline & HOMA & 0.38 & 0.0001 & & & & & & & \\
\hline & Insulin & 0.31 & 0.0004 & & & & & & & \\
\hline & Glucose & 0.41 & 0.0001 & & & & & & & \\
\hline & $\mathrm{BMI}$ & 0.37 & 0.0001 & & & & & 0.23 & 0.0444 & 0.01 \\
\hline \multirow[t]{5}{*}{ E-selectin } & $\mathrm{HbA1c}$ & 0.49 & 0.0001 & 0.001 & 0.25 & 0.0299 & & & & \\
\hline & HOMA & 0.36 & 0.0001 & 0.01 & 0.36 & 0.0015 & 0.01 & 0.28 & 0.0150 & \\
\hline & Insulin & 0.31 & 0.0006 & & 0.37 & 0.0011 & & 0.27 & 0.0172 & \\
\hline & Glucose & 0.40 & 0.0001 & & & & & 0.24 & 0.0350 & \\
\hline & BMI & 0.35 & 0.0001 & & 0.23 & 0.0456 & & 0.32 & 0.0041 & 0.01 \\
\hline
\end{tabular}


HbA1c and with HOMA, after one year, at stepwise regression, ICAM-1 and E-selectin correlated only with HOMA, indicating that after a significant weight loss, differences between NGT, IGT and T2DM disappear, and only insulin resistance still affects these molecules. Finally, at stepwise regression, changes in the three molecules only correlated with change in BMI. These findings point to an important role of obesity, and agree with the findings of Bagg et al. (10); in agreement with our data, they and others found that improvements in $\mathrm{HbAlc}$ are not associated with changes in ICAM, E-selectin and ET-1 (9, 10). In our study, body fat distribution changed in a manner similar to body weight, as assessed by waist circumference, and therefore we cannot rule out an additional role of fat distribution in the change in ICAM-1, E-selectin and ET-1, in view of the relationship between endothelial dysfunction and fat distribution (6).

With substantial slimming, triglycerides decrease significantly (11), and this might be one of the reasons why ET-1 levels decrease; tumour necrosis factor- $\alpha$ (TNF- $\alpha$ ) is produced in the adipocyte (21), and is able to directly stimulate ICAM and E-selectin release from endothelial cells (22); since substantial slimming is accompanied by a significant decrease in circulating TNF- $\alpha$ (23), it is possible that this is responsible for the decrease in ICAM and E-selectin levels.

The limitation of our study is that we analysed ICAM-1, ET-1 and E-selectin only under basal conditions and after 1 year, and therefore we cannot answer the question of a possible time-relation between change in BMI, insulin, HbA1c and glucose, and change in the three molecules under study.

We conclude that these molecules are affected by body weight, insulin resistance and abnormal glucose metabolism; all these features are present in both obesity and T2DM, and decrease after weight loss (23-25); when weight loss is substantial, only insulin resistance still affects these molecules, while if only glucose metabolism is improved, these molecules do not decrease. The practical implication of this study is that significant and durable weight reduction in obese subjects, especially when complicated by diabetes, not only improves glucose metabolism, that can be obtained also through diet and no change in body weight, but also reduces the long-term cardiovascular risk factor represented by raised circulating levels of ICAM, E-selectin and ET-1.

\section{Acknowledgements}

The authors wish to thank Monica Ferraroni, Università degli Studi di Milano, Italy, for reviewing the statistical analysis of the study. This research was supported by Grant FIRST 2002 from Università degli Studi di Milano, from Ministero della Salute (grant RF 199/02), and from Ministero dell'Università e della
Ricerca Scientifica e Tecnologica 2002 (grant 2002064582_003).

\section{References}

1 De Vriese AS, Verbeuren TJ, Van de Voorde J, Lameire NH \& Vanhoutte PM. Endothelial dysfunction in diabetes. British Journal of Pharmacology $2000130963-974$.

2 Steiner M, Reinhardt KM, Krammer B, Ernst B \& Blann AD. Increased levels of soluble adhesion molecules in type 2 diabetes mellitus are independent of glycaemic control. Thrombosis and Haemostasis 199472 979-984.

3 Bonetti PO, Lerman LO \& Lerman A. Endothelial dysfunction. A marker of atherosclerotic risk. Arteriosclerosis, Thrombosis and Vascular Biology 200323 168-175.

4 Hattori Y, Kasai K, Nakamura T, Emoto T \& Shimoda S. Effect of glucose and insulin on immunoreactive endothelin-1 release from cultured porcine aortic cells. Metabolism 199140 165-170.

5 Ferri C, Pittoni V, Piccoli A, Laurenti O, Cassone MR, Bellini C et al. Insulin stimulates endothelin-1 secretion from human endothelial cells and modulates its circulating levels in vivo. Journal of Clinical Endocrinology and Metabolism $1995 \mathbf{8 0} 829-835$.

6 Arcaro G, Zamboni M, Rossi L, Turcato E, Covi G, Armellini F et al. Body fat distribution predicts the degree of endothelial dysfunction in uncomplicated obesity. International Journal of Obesity and Related Metabolic Disorders 199923 936-942.

7 Steinberg HO, Chaker H, Leaming R, Johnson A, Brechtel G \& Baron AD. Obesity/insulin resistance is associated with endothelial dysfunction. Implications for the syndrome of insulin resistance. Journal of Clinical Investigation $1996972601-2610$.

8 Piatti PM, Monti LD, Conti M, Baruffaldi L, Galli L, Pontiroli AE et al. Hypertriglyceridemia and hyperinsulinemia are potent inducers of endothelin-1 release in humans. Diabetes $1996 \mathbf{4 5}$ 316-321.

9 Yudkin JS, Panahloo A, Stehouwer C, Emeis JJ, Bulmer K, Mohamed-Ali V et al. The influence of improved glycaemic control with insulin and sulphonylureas in acute phase and endothelial markers in type II diabetic subjects. Diabetologia $2000 \mathbf{4 3}$ 1099-1106.

10 Bagg W, Ferri C, Desideri G, Gamble G, Ockelford P \& Braatvedt GD. The influences of obesity and glycemic control on endothelial activation in patients with type 2 diabetes. Journal of Clinical Endocrinology and Metabolism 2001 86 5491-5497.

11 Pontiroli AE, Pizzocri P, Librenti MC, Vedani P, Marchi M, Cucchi E et al. Laparoscopic adjustable gastric banding for the treatment of morbid (grade 3) obesity and its metabolic complications: a threeyear study. Journal of Clinical Endocrinology and Metabolism 2002 87 3555-3561.

12 Anonymous. Physical status: the use and interpretation of anthropometry: report of a WHO expert committee. WHO Technical Report Series $1995 \mathbf{8 5 4} 1-452$.

13 National Institutes of Health, Clinical guidelines on the identification, evaluation, and treatment of overweight and obesity in adults - the evidence report. Obesity Research 19986 (Suppl 2) 51S-209S.

14 Pontiroli AE, Pizzocri P, Giacomelli M, Marchi M, Vedani P, Cucchi E et al. Ultrasound measurement of visceral and subcutaneous fat in morbidly obese patients before and after laparoscopic adjustable gastric banding: comparison with computerized tomography and with anthropometric measurements. Obesity Surgery 200212 648-651.

15 Alberti KGMM \& Zimmet PZ. Definition, diagnosis and classification of diabetes mellitus and its complications. Part 1: diagnosis and classification of diabetes mellitus - provisional report of a WHO consultation. Diabetic Medicine 1998 15 539-553.

16 The DCCT Research Group, Feasibility of centralized measurements of glycated hemoglobin in the Diabetes Control and Complications Trial: a multicenter study. Clinical Chemistry 198733 $2267-2271$. 
17 Kanai H, Hirakata H, Nakayama M, Nagashima A \& Fujishima M. Minimal daily variations of plasma and urinary endothelin-1 in healthy subjects. Clinical Nephrology $1996 \mathbf{4 6} 353-354$.

18 Blann AD \& Lip GY. Endothelial integrity, soluble adhesion molecules and platelet markers in type 1 diabetes mellitus. Diabetic Medicine 199815 634-642.

19 Rothlein R, Mainolfi EA, Czajkowski M \& Marlin SD. A form of circulating ICAM-1 in human serum. Journal of Immunology 1991 $1473788-3793$.

20 Matthews DR, Hosker JP, Rudenski AS, Naylor BA, Treacher DF \& Turner RC. Homeostasis model assessment: insulin resistance and $\beta$-cell function from fasting plasma glucose and insulin concentrations in man. Diabetologia $1985 \mathbf{2 8} 412-419$.

21 Hotamisligil GS \& Spiegelman BM. Tumor necrosis factor alpha: a key component of the obesity-diabetes link. Diabetes $1994 \mathbf{4 3}$ 1271-1278.

22 Schmidt A, Goepfert C, Feitsma K \& Buddecke E. Lovastatinstimulated superinduction of E-selectin, ICAM-1 and VCAM-1 in
TNF-alpha activated human vascular endothelial cells. Atherosclerosis $200216457-64$.

23 Ziccardi P, Nappo F, Giugliano G, Esposito K, Marfella R, Cioffi M et al. Reduction of inflammatory cytokine concentrations and of endothelial function in obese women after weight loss over one year. Circulation $2002105804-809$.

24 Ferri C, Desideri G, Valenti M, Bellini C, Pasin M, Santucci A et al. Early upregulation of endothelial adhesion molecules in obese hypertensive men. Hypertension 199934 568-573.

25 Chen NG, Holmes M \& Reaven GM. Relationship between insulin resistance, soluble adhesion molecules, and mononuclear cell binding in healthy volunteers. Journal of Clinical Endocrinology and Metabolism 199984 3485-3489.

Received 14 July 2003

Accepted 28 October 2003 